\title{
Lung function tests and risk factors for pneumonia in adults with chickenpox
}

\author{
A H Mohsen, R J Peck, Z Mason, L Mattock, M W McKendrick
}

\begin{abstract}
Background-Varicella is 25 times more likely to be complicated by pneumonia in adults than in children. Data on changes in lung function following pneumonia are limited. This study was undertaken to describe the epidemiological factors associated with pneumonia and to investigate lung function up to 1 year following chickenpox.

Methods-Thirty eight consecutive suitable patients admitted to a university hospital were enrolled in the study; 19 had pneumonia and 19 did not. Epidemiological data and density of rash were recorded, spirometric tests were performed, and carbon monoxide transfer factor was measured.

Results-Varicella pneumonia was associated with the presence of respiratory symptoms $(p=0.006)$, current smoking $(p=0.003)$, and history of close contact $(p=0.009)$. There was a trend towards patients with pneumonia having a more severe rash. No association was observed between pneumonia and age or sex. Current smokers had a higher mean number of spots than non-smokers $(p=0.005)$. Carbon monoxide transfer factor at hospital discharge was reduced in 27 patients $(71 \%)$, more markedly in the group with pneumonia $(p=0.009)$. Nine patients (seven with pneumonia) still had a reduced carbon monoxide transfer factor (mean reduction $36 \%$ ) at 12 months.

Conclusion-Chickenpox may result in a defect in carbon monoxide transfer factor for at least a year after acute illness.
\end{abstract} (Thorax 2001;56:796-799)

Keywords: varicella pneumonia; chickenpox; lung function; carbon monoxide transfer factor

Received 20 November 2000 Returned to authors 14 March 2001

Revised version received

23 May 2001

Accepted for publication

4 July 2001

The incidence of chickenpox in adults has more than doubled in England and Wales over the last two decades and has been parallelled by

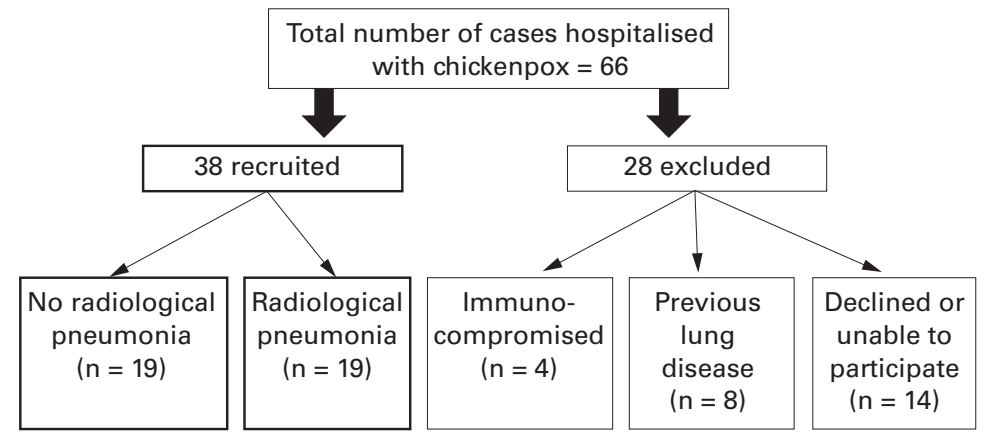

Figure 1 Selection of patients for study. an increase in admissions to hospital and mortality. ${ }^{1-3}$ A similar trend has also been observed in the USA. ${ }^{4}$ Varicella pneumonia is the most common serious complication of chickenpox infection in adults ${ }^{56}$ with a reported incidence of $16-33 \%^{7}$ and mortality of up to $20 \%,{ }^{8}$ although the incidence and mortality figures are probably high due to selective reporting. Early diagnosis and appropriate treatment is likely to reduce morbidity and mortality related to these complications. Chickenpox can leave permanent radiological changes on the chest radiograph-mainly speckled calcification-which may develop up to 7 years after recovery, ${ }^{8-10}$ but there are few data on the long term effects on lung function. There is evidence to suggest that bacterial pneumonia in childhood can cause a reduction in lung function in later life, typical changes including a restrictive ventilatory defect with a reduction in static lung volumes. ${ }^{11}$

The purpose of this study was to identify the clinical and epidemiological characteristics associated with pneumonitis and to investigate prospectively lung function up to 1 year following chickenpox.

\section{Methods}

Immunocompetent adults with a clinical diagnosis of chickenpox admitted to the North Trent Department of Infection and Tropical Medicine between June 1997 and November 1999 were studied prospectively (fig 1). Patients were excluded if they had a history of prior lung disease. Patients were referred to hospital for further assessment by the general practitioners or accident and emergency officers who were not aware of the study.

The characteristics collected at the time of admission included age, sex, history of contact with chickenpox (within the previous 2 months, with type of contact), smoking history (current, past, never smoked, number of cigarettes per day), and current pregnancy. The incubation period was calculated by taking the first day of contact with the source patient (after rash had occurred) to the day of developing rash. Respiratory symptoms of breathlessness, chest pain, chest tightness, and cough were recorded.

The density of the rash was recorded by a single observer who counted the number of spots within a "chest box" defined as the area between parallel vertical lines through the nipples and horizontal lines at the levels of the clavicle and nipples. ${ }^{12}$ Patients were considered to have chickenpox pneumonia if the chest radiograph on admission showed changes consistent with acute infection (diffuse nodular/ reticular changes). A single observer (consultant radiologist), blinded to the diagnosis, 
Table 1 Demographic data of cohort and risk factors for developing pneumonia

\begin{tabular}{llll}
\hline & Pneumonia $(n=19)$ & No pneumonia $(n=19)$ & Odds ratio (OR), $p$ value \\
\hline Sex (F:M) & $7: 12$ & $7: 12$ & $\mathrm{NS}$ \\
Mean age (years) & 29 & 28 & $\mathrm{p}=0.51^{\star}$ \\
Mean no of spots in chest box & 129 & 91 & $\mathrm{p}=0.13^{\star}$ \\
Mean hospital admission (days) & 6.4 & 3.1 & $\mathrm{p}=0.012^{\star}$ \\
Respiratory symptoms at presentation & 18 & 9 & $\mathrm{OR}=18(95 \%$ CI 2 to 165$) ; \mathrm{p}=0.006 \dagger$ \\
Smoker & 16 & 6 & OR=11.5 (95\% CI 3.3 to 40.4$) ; \mathrm{p}=0.003 \dagger$ \\
Any contact with chickenpox & 16 & 10 & OR=4.8 (95\% CI 1.0 to 22.1); $\mathrm{p}=0.08 \dagger$ \\
History of contact with own child & 14 & 5 & $\mathrm{OR}=7.8(95 \%$ CI 1.8 to 33.2$) ; \mathrm{p}=0.009 \dagger$ \\
Pregnancy & 2 (both 3rd & $2(1$ st and 3rd trimester) & \\
& trimester) & & \\
Admission to ITU & 3 & 0 &
\end{tabular}

* Non-parametric Mann-Whitney U test; †Fisher exact test.

reviewed all the chest radiographs. Duration of admission and complications were recorded.

Respiratory function tests were performed on discharge, at 2 months and, if abnormal, every 3 months until they became normal or up to 1 year from the acute infection. The tests included forced expiratory volume in 1 second $\left(\mathrm{FEV}_{1}\right)$, forced vital capacity (FVC), $\mathrm{FEV}_{1} /$ FVC ratio (Vitalograph), and single breath carbon monoxide transfer factor (TLCO) (Sensormedics Vmax 22). Smokers were asked not to smoke on the day of the tests.

The results of lung function tests in the patient group were compared with the predicted normal values for those of comparable age, sex, height, and weight. ${ }^{13}$ The methods used for calculating predicted TLCO and lung volumes were based on the European guideline. ${ }^{13}$ TLCO and $\mathrm{FEV}_{1}$ measurements were considered abnormal if they were below $75 \%$ of predicted normal values. ${ }^{14} 15$ The percentage of the reference predicted normal value was used to compare the different groups of patients. All patients who had a TLCO measurement had a normal haemoglobin and TLCO values were corrected for alveolar volume to produce the transfer coefficient (KCO). The $\mathrm{FEV}_{1} / \mathrm{FVC}$ ratio was considered abnormal if it was less than $70 \%$ of the predicted normal value. ${ }^{15}$

DATA ANALYSIS

Patients with radiological pneumonia were compared with patients with a normal chest radiograph at presentation. Odds ratios (OR) were calculated for each comparison and Fisher's exact test was used to assess significance. A non-parametric test (Mann-Whitney $U$ test) was used to compare age and difference in TLCo between the two groups. To examine the effect of smoking on TLCO, the numbers of smokers in normal and reduced TLCO groups were counted in patients with complete follow up data. The OR of reduced TLCO in patients with pneumonia versus patients with a normal chest radiograph was calculated by logistic regression and corrected for the presence of smoking.

The study was approved by the local ethics committee and all patients gave written informed consent.

\section{Results}

Sixty six patients with chickenpox were admitted to the department during the period of study; 38 fulfilled the entry criteria and agreed to participate, $95 \%$ of whom were white. All those enrolled in the study had a chest radiograph. The demographic and epidemiological details of the 38 patients with and without pneumonia are shown in table 1 .

The mean (SD) age of the 38 patients was 29 (7) years with a male to female ratio of $1.7: 1$. Twenty six patients $(67 \%)$ gave a history of contact with chickenpox, the incubation period being 15 (6) days. Twenty seven patients (71\%) had one or more respiratory symptoms on admission which started within a median of 1 day (range -2 to 5 days) from onset of the rash. Of the 11 patients $(29 \%)$ with no respiratory symptoms on admission, only one had an abnormal chest radiograph. The presence of new respiratory symptoms in patients with chickenpox was 95\% sensitive in identifying those with radiological pneumonia but only $53 \%$ specific.

Current smokers had a higher mean number of chickenpox spots in the "chest box" (138, median 150) compared with non-smokers (71, median 65), $p=0.009$. Two patients developed cerebellar ataxia which resolved spontaneously within a few days. Three patients required admission to ITU, all female smokers one of whom was in the third trimester of pregnancy; two had caught infection from their own child. All patients with pneumonia were treated with acyclovir, initially intravenously, for 7 days. Eighteen patients $(95 \%)$ without lung involvement received acyclovir. There were no deaths. A repeat chest radiograph was available for 16 of the 19 patients with radiological pneumonia; 14 normalised, one woman developed diffuse micronodular calcifications (2 months after infection), and one still had small soft nodules at 4 months. Both patients had severe changes on their initial radiograph.

The FVC was reduced at discharge in eight patients ( $21 \%$; five with pneumonia). This normalised during follow up in five patients, two were lost to follow up after the second test, and in one the FVC was $74 \%$ of the predicted value at 12 months. The $\mathrm{FEV}_{1}$ was reduced at discharge in 14 patients ( $37 \%$; nine with pneumonia); 11 normalised at follow up, two were lost to follow up, and one achieved an $\mathrm{FEV}_{1}$ of $74 \%$ of the predicted value. The $\mathrm{FEV}_{1} / \mathrm{FVC}$ ratio was reduced in seven patients $(18 \%)$; three normalised, one was lost to follow up, and three continued to have abnormal results.

Carbon monoxide transfer factor was reduced at discharge in 27 patients $(71 \%), 16$ $(84 \%)$ with pneumonia and $11(57 \%)$ with a normal chest radiograph (fig 2A). Seven 

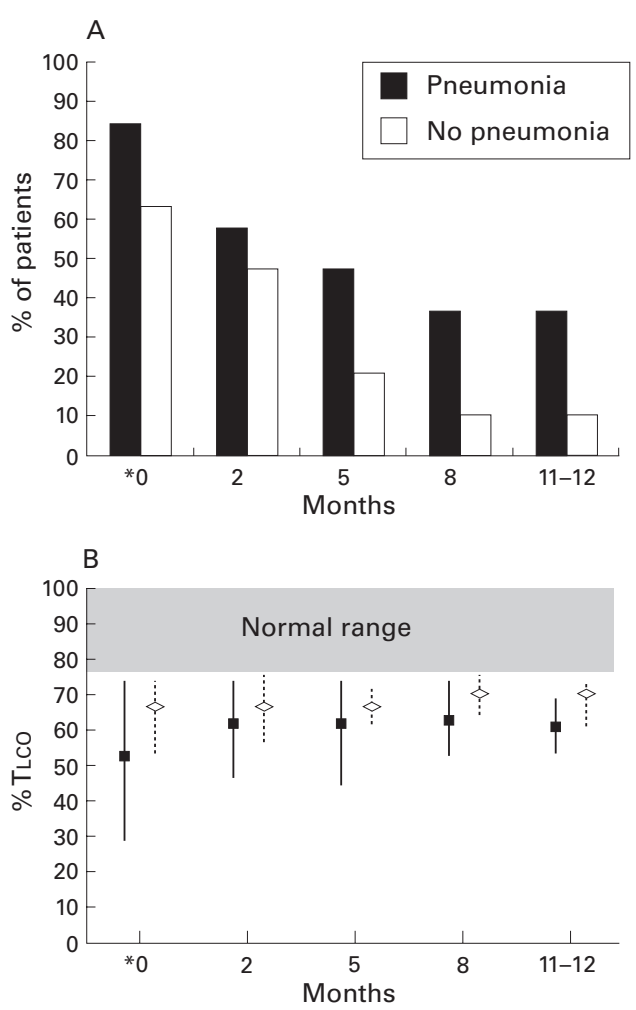

Figure 2 (A) Percentage of patients remaining with reduced carbon monoxide transfer factor (TLCO) in patients with $(n=19)$ and without $(n=19)$ pneumonia during follow up. Of those who presented with pneumonia, $37 \%$ still had reduced TLCO at 12 months compared with $10.6 \%$ of those with no pneumonia. (B) Degree of impairment in patients with persisting reduced TLCO for each of the groups (mean and range). The number of patients with persisting abnormalities in each group are shown in $(A) .{ }^{*} O n$ hospital discharge 18 patients reached normal range by 12 months; 11 patients were lost to follow up during the study period.

normalised during follow up, but nine patients (seven with pneumonia) still had a reduced transfer factor at 12 months (mean $36 \%$ ) as shown in fig $2 \mathrm{~B}$. On discharge from hospital, patients with pneumonia had a greater reduction in TLCO than those with a normal chest radiograph $(p=0.003)$. Complete data on TLCO were available on 27 patients, 10 smokers (seven reached normal levels) and 17 nonsmokers (11 reached normal levels). The frequency of smoking was not significantly different between the two group $(p=0.77)$. The OR for reduced TLCO in the pneumonia group was 8.75 (95\% CI 0.88 to $86.6, p=0.06$ ) compared with the group with a normal chest radiograph. This OR did not reach significance because of the small number of patients in the study. Nevertheless, the OR did allow the interaction of smoking to be examined. After correcting for smoking, the OR was 12.99 (95\% CI 0.77 to $217, \mathrm{p}=0.07$ ) for reduced TLCO in the pneumonia group.

\section{Discussion}

Chickenpox in healthy adults has a 25 fold greater risk of complications than in a child. ${ }^{16}$ Hospitalised patients are clearly a selected population and most in the UK are referred by general practitioners or $\mathrm{A} \& \mathrm{E}$ departments because of the severity of the infection. In this study $73 \%$ of suitable patients admitted to the department agreed to participate in the study, $50 \%$ of whom had radiological pneumonia. It is probable that subclinical lung involvement in chickenpox is common but, for the purposes of this study, patients were defined as having pneumonia only if they had radiological changes. The study was not designed to assess the incidence of pneumonia in chickenpox.

We found that a history of contact with a patient's own child with chickenpox was a risk factor for pneumonia (OR=7.8), an association that has not been shown previously. There was also an association between any recognised contact and development of pneumonia $(\mathrm{OR}=4.8)$. This could be a consequence of these patients having a closer contact with the index case and therefore receiving a larger "infecting dose" with an enhanced primary viraemia; it has previously been demonstrated that children who get chickenpox from siblings usually have a worse disease with more spots. ${ }^{17}$ Smoking was associated with increased incidence of pneumonia in this series $(\mathrm{OR}=11.5)$, as has been previously noted. ${ }^{14}$

Deciding who should be referred to hospital can be difficult for the general practitioner. We found a strong correlation between pneumonia and new respiratory symptoms (breathlessness, chest pain, chest tightness, and cough), symptomatic patients having an 18 fold higher risk of radiological pneumonia. Referral to hospital for formal evaluation should definitely be recommended for patients with chickenpox who develop new respiratory symptoms. Patients with pneumonia also had a trend towards having an increased number of skin spots $(\mathrm{p}=0.13)$ but, interestingly, smokers were also found to have more skin spots than nonsmokers $(p=0.009)$, an association not previously reported. It is possible that smokers have more spots because of an enhanced primary viraemia secondary to the effects of smoking on the nasal mucosa, and this predisposes to pneumonia. Unfortunately, there were insufficient patients in this study to perform a multivariate analysis and to determine whether the number of spots and smoking were independent variables. A previous report has shown that smoking renders human alveolar macrophages more susceptible to infection by herpes viruses $^{18}$ which could be relevant pathogenetically, although this requires further study. The number of pregnant women in this series was small but pregnancy probably increases the risk of developing pneumonia, particularly in the third trimester, because of changes in the immune system. ${ }^{19}{ }^{20}$ Severe disease requiring admission to ITU was observed only in women in this series.

Acyclovir has been shown to be of clinical benefit to adults receiving it within 24 hours of the onset of rash. ${ }^{21}$ However, there has been no study with sufficient power to address whether antiviral therapy will prevent complications such as pneumonia. There is indirect evidence to suggest that intravenous acyclovir may be of benefit in treating chickenpox pneumonia and its usage has been recommended. ${ }^{3}$ Varicella zoster immunoglobulin is expensive and in short supply and its use is therefore restricted. 
The use of acyclovir as prophylaxis/treatment during the incubation period in chickenpox has been shown to prevent or modify illness, ${ }^{22}$ although it is not currently licensed for this indication in the UK but this intervention may be used more in the future. Recent evidence suggests that varicella vaccine is effective in preventing or modifying severity if used within 3 days and possibly even up to 5 days of exposure $^{23}{ }^{24}$; the US Advisory Committee on Immunization Practices includes a recommendation about administration of varicella vaccine after exposure. ${ }^{25}$ It is contraindicated in pregnancy and at present the varicella vaccine is not licensed in the UK. The result of this study may contribute to the argument for the implementation of vaccination as part of the childhood vaccination programme.

A Medline search of the literature revealed only two published studies on respiratory function in chickenpox, and these included only 14 patients with chickenpox pneumonia. ${ }^{14}{ }^{26} \mathrm{Nei}-$ ther of these studies indicated whether the patients studied had any history of previous pneumonia or immunosuppression. The mean (SD) age of 33 (8) years in the study by Bocles et $a l^{26}$ was higher than in this study (29 (7) years). Only one of their patients was followed for as long as a year; six patients were followed for an average of 2-4 months. In the study by Ellis et $a l^{14} 20$ patients were studied (five with pneumonia); none had severe disease and follow up was only for 2 months. In our study a defect in TLCO was observed at hospital discharge in patients with and without pneumonia, as was also noted in the study by Ellis et al. ${ }^{14}$ However, the reduction in TLCO was greater in the pneumonia group $(\mathrm{p}<0.009)$. It is of interest that patients with pneumonia in the present study showed no evidence of further improvement beyond 5 months from discharge. Bocles et al studied four patients with possible chickenpox pneumonia retrospectively and demonstrated a defect of diffusion up to 8 years after the acute illness, ${ }^{26}$ which raises the possibility that the prospectively observed changes noted in our study might be permanent.

We considered the possibility that smoking had influenced our findings. There were equal numbers of smokers in the patient groups with normal and reduced TLCO. Furthermore, multivariate analysis confirmed that the OR for pneumonia as a predictor of reduced TLCO increased after correction for smoking. There was therefore no evidence to suggest that the observed decrease in TLCO was attributable to smoking.

The temporary changes in $\mathrm{FVC}$ and $\mathrm{FEV}_{1}$ are probably the result of diffuse inflammation of lung tissue affecting patients with and without radiological evidence of pneumonia. This indicates a reduction in lung volumes as the $\mathrm{FEV}_{1}: \mathrm{FVC}$ ratio was normal in $92 \%$ of patients on their initial lung function tests. These abnormalities were observed in smokers and nonsmokers and indicate that chickenpox may be associated with a restrictive lung disease pattern in the acute and recovery phase, as was noted by
Bocles et $a l^{26}$ but not by Ellis et al. ${ }^{14}$ There was no evidence to suggest that chickenpox causes small airways disease in the recovery period.

In conclusion, we have shown that predictors of pneumonia complicating chickenpox include the presence of new respiratory symptoms, smoking, and close contact with the source of infection. Chickenpox may result in a defect in the carbon monoxide transfer factor for at least 1 year after acute illness, more commonly in patients with radiological pneumonia, and may also be associated with a temporary restrictive lung defect.

The authors are grateful to Dr Stephen Gordon, honorary clinical senior lecturer in medicine, Dr Wilf Yeo, senior lecturer in medicine and Dr Robert Read, reader in infectious diseases, for helpful comments.

No financial support was obtained for this study.

1 Miller E, Vurdien J, Farrington P. Shift in age in chickenpox. Lancet 1993;341:308-9.

2 Joseph C, Noah ND. Epidemiology of chickenpox in England and Wales, 1967-85. BMF 1988;296:308-9.

3 Wilkins EGL, Leen CLS, McKendrick MW, et al. Management of chickenpox in adults. F Infect 1998;1:49-58.

4 Gray GC, Palinkas LA, Kelley PW. Increasing incidence of varicella hospitalisations in the United States Army and Navy personnel: are today's teenagers become more susceptible? Should recruits be vaccinated? Pediatrics 1990; 86:867-73.

5 Guess HA, Broughton DD, Melton LJ, et al. Chickenpox hospitalizations among residents of Olmsted Country, Minnesota, 1962 through 1981. Am f Dis Child 1984;138: 1055-7.

6 Poltkin SA. Clinical and pathogenetic aspects of varicellazoster. Postgrad Med $\mathcal{f} 1985 ; 61$ (Suppl 4):7-14

7 Anonymous. Chicken-pox pneumonia. BMF 1967;4:438

8 Knyvett $\mathrm{AF}$. The pulmonary lesions in chickenpox. $Q \mathcal{F} \mathrm{Med}$ 1966;139:313-23.

9 Anonymous. Chicken-pox pneumonia. BMF 1966;2:965-6. Raider L. Calcification in chickenpox pneumonia. Chest 1971;60:504-7.

11 Johnston IDA, Strachan DP, Anderson HR. Effect of pneumonia and whooping cough in childhood on adult lung function. $N$ Engl f Med 1998;338:581-7.

12 Dunkle LM, Arvin AM, Whitley RJ, et al. A controlled trial of acyclovir for chickenpox in normal children. $N$ Engl f Med 1991;325:1539-44.

13 Quanjer Ph H, Tammeling GJ, Cotes JE, et al. Lung volumes and forced ventilatory flows. Eur Respir F 1993;6(Supp 16): 4-40.

14 Ellis EM, Neal KR, Webb AK. Is smoking a risk factor for pneumonia in adults with chickenpox? BMF 1987;294: 1002 .

15 Knuddson RJ, Kaltenborn WT, Burrows B. The effects of cigarette smoking and smoking cessation on carbon monoxide diffusing capacity of the lung in asymptomatic subjects. Am Rev Respir Dis 1989;140:645-51.

16 Centre for Disease Control. Varicella-zoster immune globuin for the prevention of chickenpox. MMWR 1984;33:8490.

17 Ross A H, Lechner E, Reitman G. Modification of chickenpox in family contacts by administration of gamma globulin. $N$ Engl f Med 1962;267:369-76.

18 Rose RM, Wasserman AS, Weiser WY, et al. Deficient responses of pulmonary macrophages from healthy smokers to antiviral lymphokines in vitro. F Infect Dis $1986 ; 154$ : 611-8.

19 Rogerson SJ, Beeching NJ. Chickenpox: an association with

pregnancy. Thorax 1990;45:239.
20 Esmonde TF, Herdman G, Anderson G. Chickenpox pneumonia: an association with pregnancy. Thorax 1989; p4:812-5.

21 Wallace MR, Bowler WA, Murray NB, et al. Treatment of adult varicella with oral acyclovir: a randomised, placeboadult varicella with oral acyclovir: a randomised, plac

22 Lin TY, Huang YC, Ning HC, et al. Oral acyclovir prophylaxis of varicella after intimate contact. Pediatr Infect Dis $\mathcal{F}$ 1997;16:1162-5.

23 Watson B, Seward J, Yang A, et al. Postexposure effectiveness of varicella vaccine. Pediatrics 2000;105(1 Pt 1):84-8.

24 Asano Y, Nakayama H, Yazaki T, et al. Protection against varicella in family contacts by immediate inoculation with varicella vaccine. Pediatrics 1977;59:3-7.

25 Advisory Committee on Immunisation Practices (ACIP). Prevention of varicella: update recommendation of the Advisory Committee on Immunisation Practices (ACIP). MMWR 1999;48:2.

26 Bocles JS, Ehrenkranz NJ, Marks A. Abnormalities of respiratory function in varicella pneumonia. Ann Intern Med 1964;60:183-95. 